\title{
Effect of new bedding material on morpho-biochemical and immunological factors of gosling blood
}

\author{
Vladimir Sofronov, Ramil Fayzrakhmanov, Nadezhda Danilova* and Elena Kuznetsova \\ Kazan State Academy of Veterinary Medicine named after N.E. Bauman, Kazan, 420029, Republic of Tatarstan, Russia
}

\begin{abstract}
The purpose of research and production experiment in the conditions of Akhmetov farm located in the Vysokogorsky district of the Republic of Tatarstan was to study the harmlessness of new sanitary bedding material and the effect on gosling broilers. The experiment evidenced that a new sanitary bedding material made of woodworking waste, thermochemically and biotechnologically treated, does not have an irritating effect on the skin and mucous membranes of the eye, nor does it provoke allergic reactions in rabbits, but, on the contrary, improves certain morphobiochemical and immunological factors of gosling blood. Ultimately, this resulted in accelerated red blood cells by $6.94 \%$, hemoglobin $-5.20 \%$, total protein $-6.30 \%, \gamma$-globulin $-9.38 \%$, total calcium $-6.14 \%$, inorganic phosphorus $-5.31 \%$, glucose $5.38 \%$, increased phagocytic activity by $2.24 \%$, phagocytic number $-4.37 \%$, and increased number of S. Marcescens-stimulated cells through the NBT test by $5.17 \%$ compared with the control, respectively. The data obtained are consistent with the advanced livestock viability in the experiment by $1.8 \%$, as compared with the control.
\end{abstract}

\section{Introduction}

An important role in addressing food security of any country is the growth of animal husbandry, and primarily, poultry farming. This is due to a high growth rate, minimal feed costs and the possibility of easy adaptation of poultry to modern advanced technologies, which contributes to the low cost of poultry products with its high quality [1-4].

Currently, floor brooding is more widely used in industrial poultry farming, and since the litter is used throughout the brooding period, it is subjected to very high requirements. Bedding material should have a high degree of absorption, be dry, but not dusty, absorb toxic compounds from the environment and suppress the development of pathogens and fungi, etc., and, along with this, be harmless to fowls [5].

The quality of bedding material has a great effect on the indoor environment, and primarily on gas composition, dustiness and microbial pollution of the air. Despite the fact that the equipment used at modern poultry enterprises automatically ensures an optimal environment, poor quality bedding material can significantly affect its individual parameters [6-7].

Even slight deviations from the optimal brooding conditions can bring about lower efficiency of fowls. They become less resistant to natural conditions, which ultimately can cause a lot of non-communicable illnesses, and in some cases infectious diseases [8-10]. In such cases, a sharp drop in the resistance of fowls leads to a significant deterioration of livestock viability and has a negative effect on poultry production, which, finally, will have a negative impact on human health [11-12].

In contrast, high-quality and safe bedding materials allow for high fowl productivity at minimal cost. They reduce the risk and spread of various diseases, and, what is more important, minimize the use of drugs in fowl rearing. Ultimately, this will result in high-quality poultry products with minimal costs $[13,14]$.

Based on the foregoing, the study was aimed at the safety of a new sanitary bedding material and its effect on gosling broilers, namely, on individual morphobiochemical and immunological factors of blood in industrial production.

\section{Materials and methods}

A local irritating effect on the mucous membrane of the eye and irritating effect of a substance being applied onto the skin were studied pursuant to the Guidelines for the Studies of Irritating Properties and Substantiation of Maximum Permissible Concentrations of Selectively Acting Substances in the Air of Working Zone [15].

Morphological blood tests involved determining the number of red blood cells, white blood cells, hemoglobin and decoding the Arneth count using an automatic blood analyzer.

The content of protein and its fractions in blood serum was evaluated by vertical electrophoresis, and the total calcium and inorganic phosphorus, glucose and cholesterol in blood serum were analyzed on a BioChem SA automatic biochemical analyzer.

* Corresponding author: danai58@yandex.ru 
In an attempt to study nonspecific immunity in peripheral blood, the phagocytosis reaction was specified by a generally accepted method that implied calculating such indicators as phagocytic activity of neutrophils (pseudo-eosinophils in fowls), percentage of phagocytic cells of their total number and the phagocytic number that indicates the ability of neutrophils (pseudoeosinophils) to absorb microflora. The phagocytic activity of neutrophils (PA) of peripheral blood was determined in accordance with Z.E. Matusis and S.I. Pylaeva in the modification of E.A. Oleinikova, et al. A one-day culture of Staphylococcus Aureus served as the object of phagocytosis. A functional screening of phagocytes (pseudo-eosinophils) was provided through the NBT reduction test for more accurate and reliable data on phagocytosis. The number of neutrophils with diformazan deposits were counted: in the control spontaneously activated, and in the experience - with the use of S. Marcescens vaccine [16].

The effect of the target bedding material on gosling broilers was studied through the production experiment in the conditions of Akhmetov farm located in the Vysokogorsky district of the Republic of Tatarstan in July-August 2019. There were 4,500 geese of Lindov breed involved. The trial birds from a day to 35 days old were kept in a building $8 \times 60 \mathrm{~m}$ in size, transversely divided by a temporary partition into two halves. The totality of birds was divided into two groups, selected by exterior and live weight comparison:

- the first group - control (the first half of the building) was kept on raw sawdust delivered from the sawmill of the enterprise;

- the second group - experimental (the second half of the building) was kept on the sanitary bedding material. It was made of planed off wood chips and woodworking waste subjected to thermochemical and biotechnological processing (FPS 16.29.14-00168499146-2018). The target material is a dust-free, disinfected, loose mass from light to dark brown in color with a specific odor, which has a moisture capacity of $280 \%$ and a moisture content of $15 \%$.

The effect of new bedding material on the viability of trial birds from 1 to 35 days of age was experimentally studied.

\section{Results}

At the first stage, prior to being used in industrial poultry farming, an irritating effect of a new bedding material was examined when applied to the mucous membrane of the eye and skin of rabbits in accordance with generally accepted Guidelines.

A series of experiments to determine skin irritation were performed on 8 rabbits of both sexes. For that end, a day before the experiment the patches of skin $5 \times 5 \mathrm{~cm}$ were carefully trimmed on the back to the left and to the right of the spine. Plastic collars were put on the neck of rabbits to prevent licking of the test substance. The said bedding material was crushed in a laboratory mill and, slightly mixed with distilled water, applied natively as a wet mass to the clipped skin (at a rate of $20 \mathrm{mg} / \mathrm{cm}^{2}$ ) to the right of the spine. The control $-0.02 \mathrm{ml}$ of distilled water $/ \mathrm{cm}^{2}$ to the left of the spine for 4 hours, and then the applied preparation was removed with warm water and soap. After washing off, the skin reaction was recorded after 1 and 18 hours, compared with the symmetrical skin areas of the same animal where distilled water was applied. The rabbits were observed for 7 days.

During the follow-up period, there were no functional disturbances on the skin, and it did not differ from the control area. As per the severity of the irritating effect, the bedding material can be attributed to the substances that do not have an irritating effect on the skin of rabbits.

Eight rabbits of both sexes were used to define the irritating effect on the mucous membrane of the eye. For this purpose, $20 \mathrm{mg}$ of ground native suspension from the test bedding material was injected into the conjunctival sac of the left eye of the rabbits, while the right eye served as a control, into which 2-3 drops of distilled water were put. After applying crushed bedding material for 1 minute, the lacrimal channel was pressed at the inner corner of the eye. The state of the mucous membrane and the transparency of the cornea were monitored for a week.

The mucous membrane of the eyes did not swell up during the experiment. The corneas of the eyes were clean, though, with a slight hyperemia of the mucous membrane that disappeared in $1-1.5$ hours. The findings show that the said bedding material is a substance that does not cause irritation of the mucous membranes of the eyes of rabbits.

Thus, the bedding material does not have an irritating effect on the skin and does not cause irritation of the mucous membranes of the eyes of rabbits

An allergenic effect of the target bedding material was experimentally determined on 8 rabbits of both sexes, which had skin areas of $5 \times 5 \mathrm{~cm}$ previously trimmed on the back, left and right of the spine. The right side served for the application of a wet mass from the target material, and the left side served for control (distilled water). The experiment lasted 30 days, i.e., a wet mass from the said bedding material was daily applied to the clipped skin area. During this period, there were no allergic reactions, except for slight redness on day 25, which disappeared the next day the experiment was over. Hence, the said bedding material can be attributed to the substances that do not cause allergic reactions.

This conclusion was confirmed resulting from the research and production experiment that involved a sufficient number of fowls. The morphological blood factors of gosling broilers from the second experimental group managed on the target bedding material indicated that the number of eosinophils was within the physiological norm and slightly lower, as compared to the control group.

The effect of target bedding material on gosling broilers was verified through the production experiment in the conditions of Akhmetov farm located in the Vysokogorsky district of the Republic of Tatarstan in July-August 2019. It involved 4,500 geese of Lindov 
two-way crossing breed. The goslings were grown in a controlled-environment room in line with zoohygienic standards.

The feeding conditions for all trial goslings were the same and corresponded to generally accepted diets based on all-in-one feed (AF): AF-31 - for goslings from birth up to 4 weeks and AF-32 - up to 8 weeks [17].

The experiment was aimed at the effect of new bedding material on individual blood parameters and the viability of trial goslings from 1 to 35 days of age.

Blood is an important living environment for all cells, tissues and organs of animals. It supplies cells and tissues with nutrients, transfers metabolic products to eliminative organs, and performs a protective, humoral and thermoregulatory function. Since any husbandryrelated changes will inevitably be evidenced in the physiological status of birds, some individual morphological blood factors were studied. They are presented in Table 1.

Table 1. Morphological blood composition of trial goslings

\begin{tabular}{|c|c|c|}
\hline \multirow[t]{2}{*}{ Index } & \multicolumn{2}{|c|}{ Group } \\
\hline & 1 (control) & 2 (experiment) \\
\hline & \multicolumn{2}{|c|}{20 days } \\
\hline Erythrocytes, $10^{12} / 1$ & $2.53 \pm 0.13$ & $2.65 \pm 0.14$ \\
\hline White blood cells, $10^{9} / 1$ & $23.48 \pm 0.98$ & $23.57 \pm 0.95$ \\
\hline Hemoglobin, g/l & $96.68 \pm 5.87$ & $98.74 \pm 6.15$ \\
\hline \multicolumn{3}{|l|}{ Arneth count, $\%$} \\
\hline $\begin{array}{l}\text { pseudo-eosinophils: } \\
\text { segmented nuclei }\end{array}$ & $10.65 \pm 0.52$ & $11.85 \pm 0.58$ \\
\hline stab & $18.03 \pm 0.91$ & $19.24 \pm 0.82$ \\
\hline eosinophils & $5.23 \pm 0.27$ & $4.95 \pm 0.21$ \\
\hline basophils & $0.12 \pm 0.01$ & $0.15 \pm 0.01$ \\
\hline mid cells & $0.08 \pm 0.01$ & $0.09 \pm 0.01$ \\
\hline \multirow[t]{2}{*}{ lymphocytes } & $65.89 \pm 3.28$ & $63.72 \pm 3.41$ \\
\hline & \multicolumn{2}{|c|}{35 days } \\
\hline Erythrocytes, $10^{12} / 1$ & $2.74 \pm 0.15$ & $2.93 \pm 0.17$ \\
\hline White blood cells, $10^{9} / 1$ & $23.84 \pm 1.18$ & $23.85 \pm 1.08$ \\
\hline Hemoglobin, g/l & $104.67 \pm 4.82$ & $110.11 \pm 5.02$ \\
\hline \multicolumn{3}{|l|}{ Arneth count, \% } \\
\hline $\begin{array}{l}\text { pseudo-eosinophils: } \\
\text { segmented nuclei }\end{array}$ & $14.62 \pm 0.78$ & $15.43 \pm 0.79$ \\
\hline stab & $9.21 \pm 0.45$ & $9.84 \pm 0.48$ \\
\hline eosinophils & $4.12 \pm 0.21$ & $3.81 \pm 0.19$ \\
\hline basophils & $0.35 \pm 0.02$ & $0.31 \pm 0.04$ \\
\hline mid cells & $1.98 \pm 0.09$ & $2.03 \pm 0.08$ \\
\hline lymphocytes & $69.72 \pm 3.48$ & $68.58 \pm 3.41$ \\
\hline
\end{tabular}

The data shown in Table 1 suggest that the use of experimental processed sawdust as bedding material (the second experimental group) has a positive effect on individual morphological blood factors of experimental gosling broilers, unlike the control (first group) managed on raw sawdust. Thus, the number of red blood cells at the age of 20 days was $4.74 \%$ higher, and at the end $6.94 \%$ hemoglobin -2.13 and $5.20 \%$, and eosinophils was lower by 5.36 and $7.53 \%$, respectively. The number of leukocytes in trial goslings in both groups was approximately at the same level.

Thus, the target bedding material has a positive effect on goslings, which is justified by augmented number of red blood cells and hemoglobin and reduced number of eosinophils.
At the current rate of industrial poultry development biochemical blood factors are an important component of efficient production. Advanced farms with a high level of production hold periodic biochemical examinations of livestock blood in order to monitor nutrition and correct metabolism in the body of fowls. Some biochemical blood parameters of trial birds are presented in Table 2.

Table 2. Some biochemical blood parameters of trial gosling broilers

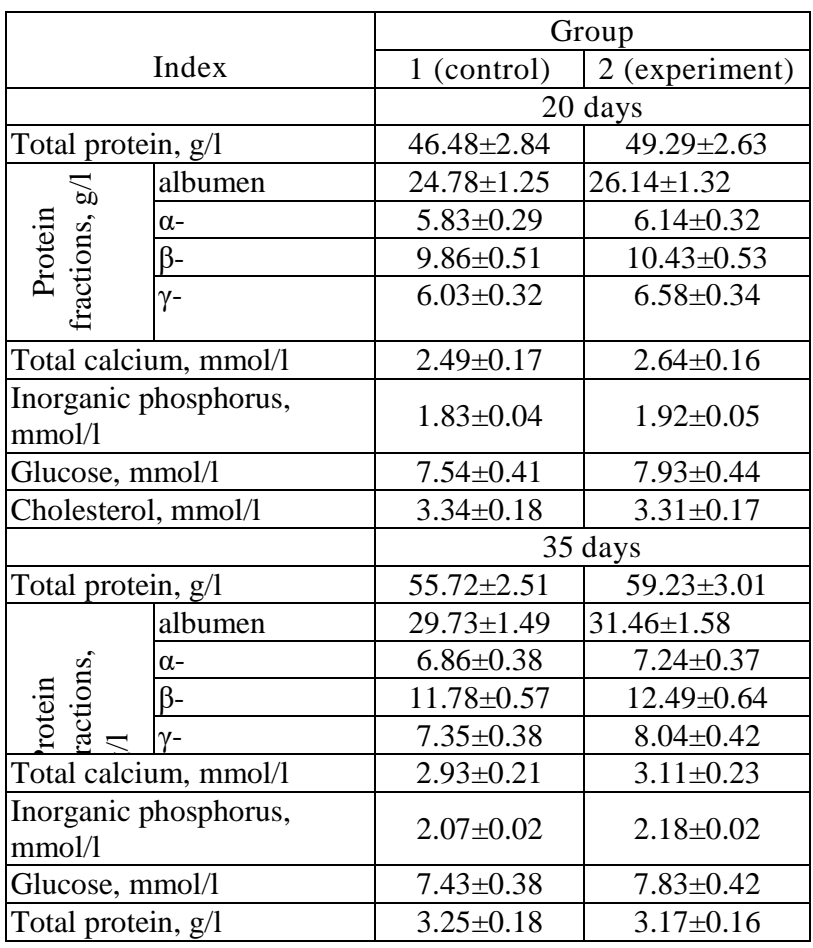

The data in Table 2 indicate that at the age of 20 days and 35 days, the total protein accelerated by 6.04 and $6.30 \%$, respectively, in the blood serum of goslings from the second experimental group, in which the processed sawdust was used as bedding material, as compared to the first control group managed on raw sawdust. The total calcium increased by 6.02 and $6.14 \%$, inorganic phosphorus - by 4.91 and $5.31 \%$, and glucose - by 5.17 and $5.38 \%$, respectively.

It is of great importance for the diagnosis of various etiological diseases to determine not only the amount of total protein, but its fractions as well. Thus, albumen supports oncotic blood plasma pressure and may indicate insufficient protein intake from food, unbalanced amino acid diet, liver, kidney, bowel diseases, etc. The amount of albumin in goslings of the experimental group after 20 days of research was 5.49 more and at the end $5.82 \%$ compared with the control.

It is also important to determine the composition of globulin fractions. The amount of $\alpha$-globulin that transport various substances in the blood plasma, from minerals to enzymes, in the goslings of the second group was 5.32 times greater than the 20-day control and 35day $-5.54 \%$. $\beta$-globulin constituting the blood coagulation system and transporting iron to the depositing organs, was 5.78 and $6.03 \%$ higher, while $\gamma$ - 
globulin associated with immunological processes in the body and including ovine antibodies was 9.12 and 9.39 $\%$ higher, respectively. Increased $\gamma$-globulins indicates a high level of the immune system of the experimental goslings, compared to the control, and is probably due to a higher microbial contamination of the bedding material by the end of the experiment throughout the room.

The serum cholesterol in all trial birds was within the physiological norm in all stages of the study.

Thus, the best husbandry conditions for experimental goslings (second group) have a positive effect on individual biochemical blood factors, as compared with control fowls (first group).

In the conditions of industrial poultry farming, a large number of birds is reared in a small room, which inevitably leads to a reduced natural resistance, and, ultimately, to lower viability. One of the informative methods for studying nonspecific immunity is to assess the phagocytic activity of neutrophils and the functional state of phagocytes. Normally, about $95 \%$ of bacteria are absorbed and destroyed by phagocytes within 2 hours. The live bacteria are found to be present in leukocytes after a thirty-minute incubation when the digestion of bacteria is impaired and the ability of phagocytes to capture bacteria is reduced, and therefore it is important to calculate not only the number of bacteria absorbed by phagocytes, but also their degradability. The functional activity of neutrophils was assessed through the NBT reduction test. Some indices of nonspecific immunity are presented in Table 3 .

Table 3. Nonspecific immunity of trial goslings

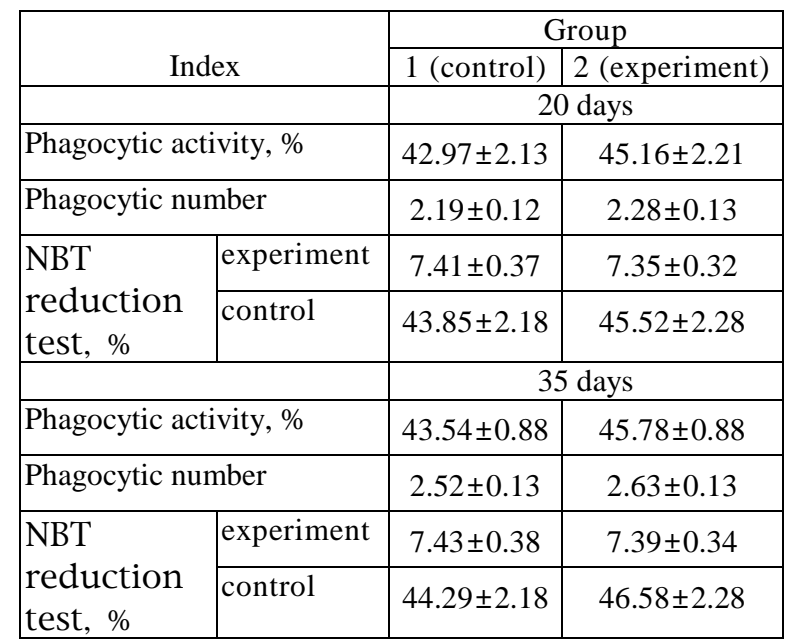

The data shown in Table 3 indicate that the use of processed bedding material helped slightly improve the phagocytic activity of neutrophils in 20-day goslings of the second experimental group, as compared to the first control group, by 2.19 and at the end $-2.24 \%$, and phagocytic numbers by 4.11 and $4.37 \%$, respectively. The second group pseudo-eosinophils had just single specimens of living bacteria, while the control had greater numbers of pseudo-eosinophils.

One of the objective methods for assessing the functional activity of neutrophils is the NBT test, based on the ability of neutrophils to phagocytose a reagent nitrosine tetrazolium, and then restore it to diformaza that is further determined under the microscope in the form of dark blue granules. The ability of pseudoeosinophils to absorb various particles, studied through the NBT test, showed a slight increase in the number of cells stimulated by $\mathrm{S}$. Marcescens vaccine (experiment): in the experimental groups on the $20^{\text {th }}$ day - to 3.81 and $35^{\text {th }}$ day - by $5.17 \%$. This indicates a higher resistance of goslings from the second experimental group in comparison with the control.

Thus, the advanced phagocytosis reaction determined through the neutrophil phagocytic activity test and confirmed by the HBT test indicates improved natural resistance of the goslings of the second experimental group, which is consistent with an increase in the safety by $1.8 \%$ compared with the first control group where goslings were bred on raw sawdust.

\section{Discussion}

Summarizing the above, the target bedding material (the second experimental group), made of planed off wood chips and woodworking waste subjected to thermochemical and biotechnological processing does not have an irritating effect on the skin, nor does it cause irritation of the mucous membranes of the eyes and allergic reactions. This was affirmed by morphological parameters of blood, showing that the number of eosinophils in experimental goslings was within the physiological norm.

The improvement of certain morpho-biochemical and immunological blood factors in the experimental goslings of the second group, in which the dust-free sawdust was used as the bedding material, can probably be due to the environment improved in the second half of the building, which contributed to the improvement of birds' health, as compared with the goslings from the first control group managed on raw sawdust.

Thus, the number of eosinophils in the goslings of the second experimental group, kept on the processed sawdust, was $7.53 \%$ lower compared to the goslings of the first control group kept on the raw sawdust. The number of red blood cells, by contrast, was higher by $6.94 \%$, hemoglobin - by $5.20 \%$, total protein in blood serum - by $6.30 \%$, $\gamma$-globulins - by $9.39 \%$, total calcium - by $6.14 \%$, inorganic phosphorus - by $5.31 \%$, glucose - by $5.38 \%$, phagocytic activity of neutrophils by $2.24 \%$, phagocytic number - by $4.37 \%$. The number of cells stimulated by S. Marcescens vaccine through the HBT reduction test increased by $5.17 \%$, respectively, which is consistent with the literature [18-19].

\section{Conclusion}

By way of conclusion, the new sanitary bedding material processed by thermochemical and biotechnological methods does not have an irritating effect on the skin, nor does it cause irritation of the mucous membranes of the eyes and allergic reactions in rabbits and, therefore, can be widely used in industrial poultry farming.

The use of new sanitary bedding material for goslings of the second experimental group helped 
increase the number of red blood cells, hemoglobin, total protein in blood serum, total calcium, inorganic phosphorus, glucose, phagocytic activity of pseudoeosinophils and phagocytic number, and increased the number of cells stimulated by $\mathrm{S}$. marcescens vaccine through the HBT reduction test, as compared to goslings of the first control group, respectively.

An improvement in the immunity in experimental goslings is evidenced by both an increase in the functional activity of pseudo-eosinophils determined in the reaction of the phagocytic activity of neutrophils and the HBT test, and an increase in $\gamma$-globulins in experimental goslings as compared to the control. An improvement in the morphological, biochemical, and immunological parameters of the blood is consistent with an increase in livestock viability by $1.8 \%$ in the experiment compared with the control, where the goslings were kept on raw sawdust.

\section{References}

1. P.L.M. van Horne, Competitiveness of the EU poultry meat sector, base year 2017: International comparison of production costs, Wageningen Economic Research, Report, p. 116 (2018)

2. David Farrell (2013). ISBN 978-92-5-108067-2 (PDF) (c) FAO. Retrieved from: www.fao.org/contact-us/

3. C.G. Scanes (2007). Retrieved from: https://www.researchgate.net/publication/5890368

4. Marie-Laure Augère-Granier, Research Service PE 644.195, 23 p. (2019)

5. K. Pelkonen, O. Hänninen, Toxicol. 122(1-2), 73-80 (1997)

6. J.L. Atencio, J.A. Fernández, A.G. Gernat, J.G. Murillo, Int. J. of Poultry Sci. 9(3), 240-243 (2010)
7. Gourav Sharma, Asma Khan, Surender Singh, Ashok Kumar Anand, Veter. World 8(10), 12191224 (2015)

8. S. Bilgili, J. Hess, J. Blake, K. Macklin, B. Saenmahayak, The J. of Appl. Poultry Res. 18(3), 583-589 (2009)

9. K. Macklin, J. Hess, S. Bilgili, R. Norton, The J. of Appl. Poultry Res. 14(2), 238-245 (2005)

10. Abdul Hafeez, S.M. Suhail, F.R. Durrani, D. and J. Sarhad, J. Agricult. 25(4), 581-586 (2009)

11. Agribusiness Consulting, Intensive broiler farming in the EU: impact on the environment, human health and animal welfare, Final report (2018)

12. Marisa Ventura da Silva ISBN 978-92-5-108067-2 (PDF) (C) FAO (2013). Retrieved from: www.fao.org/contact-us/

13. Phil Glatz and Robert Pym, ISBN 978-92-5-108067-2 (PDF) (C) FAO (2013). Retrieved from: www.fao.org/contact-us/

14. C. Burn, A. Peters, M. Day, G. Mason, Lab. Animal 40(4), 353-370 (2006)

15. Guidelines for the Studies of Irritating Properties and Substantiation of Maximum Permissible Concentrations of Selectively Acting Substances in the Air of Working Zone dated 11 August 1980, no. 2196-80 (1980)

16. G. Fremel, Immunological methods (Medicine, Moscow, 1987), pp. 376-389

17. Sh.A. Imangulov, I.A. Egorov, T.M. Okolelova, Guidelines (Sergiev Posad, 2009), 144 p.

18. V.G. Sofronov, N.I. Danilova, N.M. Shamilov, E.L. Kuznetsova, Sci. notes of Kazan State Acad. of Veter. Med. named after N.E. Bauman 227, 82-85 (2016)

19. A.I. Galiev, V.G. Sofronov, N.I. Danilova, Sci. notes of Kazan State Acad. of Veter. Med. named after N.E. Bauman 207, 114-118 (2011) 